\begin{tabular}{|c|c|}
\hline Title & MIMO Precoding Performance for Correlated and Estimated Rician Fading \\
\hline Author(s) & Shi, X iaonan (Nicole); Siriteanu, Constantin; Y oshizawa, Shingo; Miy anaga, Y oshikazu \\
\hline Citation & $\begin{array}{l}\text { グリーン回路とシステムに関する国際ワークショップ. 2011年11月4日 (金). 北海道大学情報科学研究科棟 } \\
\text { 11F17号至. 札幌市. (International Workshop on Green Circuits and Systems. Friday, } 4 \text { November, 2011. Room No.17, } \\
\text { 11th floor of G Graduate School of Information Science and Technology, Hokkaido University. Sapporo City.) }\end{array}$ \\
\hline Issue Date & 2011-11-04 \\
\hline Doc URL & http:/hdl.handle.net/2115/47542 \\
\hline Type & conference presentation \\
\hline File Information & Xiaonan_(Nicole)_Shi.pdf \\
\hline
\end{tabular}

Instructions for use 


\title{
MIMO Precoding Performance for Correlated and Estimated Rician Fading
}

\author{
Xiaonan (Nicole) Shi
}

Constantin Siriteanu Shingo Yoshizawa Yoshikazu Miyanaga

Graduate School of Information Science and Technology,

Hokkaido University, Japan

GCOE Workshop @ Hokkaido Univ. 


\section{Outline}

(1) Section 1: Introduction

(2) Section 2: Channel fading model

(3) Section 3: Precoded MIMO system model

(4) Section 4: Numerical results

(5) Section 5: Summary and conclusion 


\section{Motivation}

- Multiple-input multiple-output (MIMO) attracts research interests for next generation wireless communication systems because of its potential high capacity and reliability

- Theoretical evaluations suffer from limited practical relevance

- Important issue to exploit channel state information at transmitter (CSIT) to improve link performance 


\section{Exploit Channel State Information at Transmitter (CSIT)}

\section{Benefits}

- Allows transmitter know full/partial channel state information

- Alleviates the interference when preprocessing the data before transmission

- Provides the extra gain by using CSIT.

Issues need to work

- Perfect CSIT is not available in wireless communications

- Estimated CSIT usually relies on only independent antenna assumptions

- Practical channel environment relates to both instantaneous and statistics channel state information (CSI). 


\section{Precoded MIMO Block Diagram}

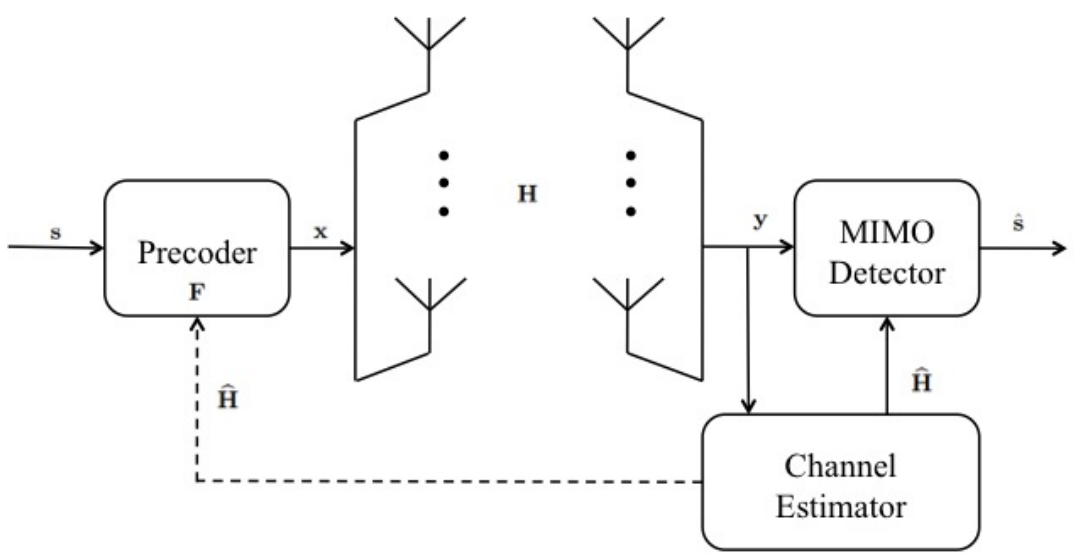




\section{Channel Model}

Channel matrix has complex-valued Gaussian-distributed elements with unit variance, and can be written as:

$$
\mathbf{H}=\mathbf{H}_{\mathrm{d}}+\mathbf{H}_{\mathrm{r}}=\sqrt{\frac{K}{K+1}} \mathbf{H}_{\mathrm{d}, \mathrm{n}}+\sqrt{\frac{1}{K+1}} \mathbf{H}_{\mathrm{r}, \mathrm{n}} .
$$

- $\mathbf{H}_{\mathrm{d}, \mathrm{n}}$ : normalized deterministic component, with $\operatorname{rank}\left(\mathbf{H}_{\mathrm{d}, \mathrm{n}}\right)=1: N_{\mathrm{T}}$

- $\mathbf{H}_{r, n}$ : normalized random component, with $\mathbf{H}_{r, n}=\mathbf{R}_{\mathrm{Rx}}^{\frac{1}{2}} \mathbf{H}_{w} \mathbf{R}_{\mathrm{Tx}}^{\frac{1}{2}}$

- Spatial correlation matrix for Tx correlated only is $\mathbf{R}_{\mathrm{H}}=\frac{1}{K+1} \mathbf{R}_{\mathrm{TX}}$, in which $\mathbf{R}_{\mathbf{T X}}$ is affected by azimuth spread (AS)

- $\mathbf{H}_{w}$ : i.i.d. channel matrix

- $K$ : Rician $K$-factor (Rician fading : $K \neq 0$, Rayleigh fading : $K=0$ ) 


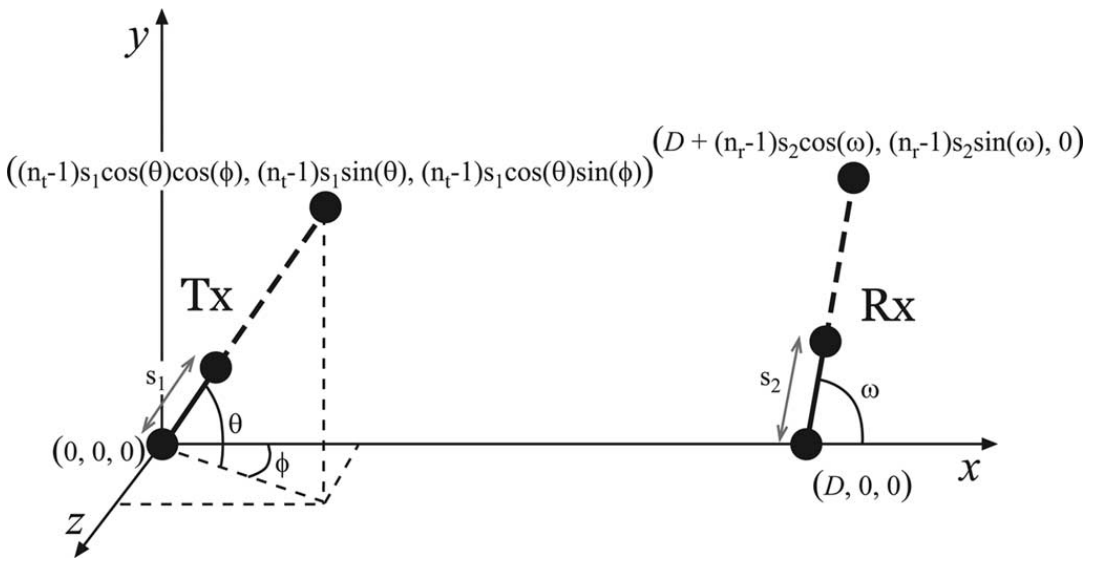




\section{WINNER II Channel Model}

- Most comprehensive available

- Measured radio channel extensively in a wide range of scenarios

- Developed statistical models for the fading based on thorough measurement (always Rician fading, never Rayleigh fading)

- Distinguished its models from other mostly theoretical models. 


\section{AS-K Statistical Distribution}

Table: Base-station AS and $K$ distribution

\begin{tabular}{|l||c|c|c|}
\hline Scenario & $\left.\mathrm{AS}^{\circ}\right]$ & $K$ & $\rho_{\chi, \psi}$ \\
\hline \hline A1: indoor office/residential & $10^{1.64+0.31 \chi}$ & $10^{0.1(7+6 \psi)}$ & -0.6 \\
\hline B1: typical urban microcell & $10^{0.40+0.37 \chi}$ & $10^{0.1(9+6 \psi)}$ & -0.3 \\
\hline B3: large indoor hall & $10^{1.22+0.18 \chi}$ & $10^{0.1(2+3 \psi)}$ & +0.2 \\
\hline C1: suburban & $10^{0.78+0.12 \chi}$ & $10^{0.1(9+7 \psi)}$ & +0.2 \\
\hline C2: typical urban macrocell & $10^{1.00+0.25 \chi}$ & $10^{0.1(7+3 \psi)}$ & +0.1 \\
\hline D1: rural macrocell & $10^{0.78+0.21 \chi}$ & $10^{0.1(7+6 \psi)}$ & +0.0 \\
\hline D2a: rural, high-speed & $10^{0.70+0.31 \chi}$ & $10^{0.1(7+6 \psi)}$ & +0.0 \\
\hline
\end{tabular}




\section{System Signal Model}

The system has $N_{T}$ transmit antennas and $N_{R}$ receive antennas. The transmitter precodes a $N_{T} \times 1$ source symbol vector $\mathbf{s}$ with a precoding matrix $\mathbf{F}$, where the precoding matrix has a size of $N_{\top} \times N_{\top}$, i.e.,

$$
\mathbf{x}=\mathbf{F s} .
$$

Then, received signals can be represented as the $N_{R}$-dimensional complex-valued vector:

$$
\mathbf{y}=\mathbf{H x}+\mathbf{n},
$$

where $\mathbf{x}$ is the $N_{\mathrm{T}}$-dimensional vector with the precoded complex-valued transmitted symbols, $\mathbf{H}$ is the channel matrix with complex-valued Gaussian distributed elements with unit variance, and the noise vector is complex-valued, zero-mean, spatially-uncorrelated, Gaussian distributed, i.e., $\mathbf{n} \sim \mathcal{N}_{\mathrm{c}}\left(0, N_{0} \mathbf{I}\right)$. 


\section{Linear Precoding Approaches}

Linear precoding is the most available for current advanced transmitter design.

Zero Forcing (ZF) Approach

- Suboptimal that less achievable in capacity

- Still can maximize the performance in some environments

\section{Minimum Mean Square Error (MMSE) Approach}

- Optimal approach that is available for precoding

- Simple to characterize the nose effect on the performance 


\section{Mismatched Zero Forcing (ZF) Precoding}

The ZF approach cancels the inter-stream interference by the following matrix:

$$
\mathbf{F}_{\mathrm{ZF}}=\left(\widehat{\mathbf{H}}^{H} \widehat{\mathbf{H}}\right)^{-1} \widehat{\mathbf{H}}^{H},
$$

where $(\cdot)^{H}$ denotes Hermitian transposition. 


\section{Mismatched Minimum Mean Square Error (MMSE) Precoding}

In order to maximum precoding interference plus noise cancellation, MMSE precoding minimizes the minimum square error between the transmitted symbols and received symbols. The precoding matrix is given by:

$$
\begin{aligned}
\mathbf{F}_{\mathrm{MMSE}} & =\arg \min _{\mathbf{F}} \mathbb{E}\left\{\|(\mathbf{H F s}+\mathbf{n})-\mathbf{s}\|_{F}^{2} \mid \mathbf{H}\right\} \\
& =\left(\widehat{\mathbf{H}}^{H} \widehat{\mathbf{H}}+N_{0} \mathbf{I}\right)^{-1} \widehat{\mathbf{H}}^{H},
\end{aligned}
$$

where $\|\cdot\|_{F}^{2}$ represents the Frobenius spectral norm of a matrix. 


\section{New Approach}

Given the estimated channel matrix, the true channel matrix can be rewritten as:

$$
\mathbf{H}=\mathbf{H}_{\mathrm{m}}+\mathbf{H}_{\mathrm{e}}
$$

where $\mathbf{H}_{\mathrm{m}}$ is given by:

$$
\mathbf{H}_{\mathrm{m}}=\mathbb{E}\{\mathbf{H} \mid \widehat{\mathbf{H}}\}=\mathbb{E}\{\mathbf{H}\}+\mathbf{R}_{\mathrm{T}}\left(\mathbf{R}_{\mathrm{T}}+N_{0} \mathbf{I}\right)^{-1}(\widehat{\mathbf{H}}-\mathbb{E}\{\widehat{\mathbf{H}}\}) .
$$

The rows of $\mathbf{H}_{\mathrm{e}}$ follow the distribution of $\mathcal{N}_{\mathrm{c}}\left(0, \mathbf{R}_{\mathrm{e}}\right)$, where

$$
\mathbf{R}_{\mathrm{e}}=\mathbf{R}_{\mathrm{T}}-\mathbf{R}_{\mathrm{T}}\left(\mathbf{R}_{\mathrm{T}}+N_{0} \mathbf{I}\right)^{-1} \mathbf{R}_{\mathrm{T}}^{H} .
$$

Then we can rewrite the received signal as:

$$
\mathbf{y}=\mathbf{H}_{\mathrm{m}} \mathbf{x}+\boldsymbol{\nu},
$$

where the new noise vector $\boldsymbol{\nu}=\mathbf{H}_{\mathrm{e}} \mathbf{x}+\mathbf{n}$ is zero-mean, complex-valued, Gaussian-distributed with correlation matrix:

$$
\mathbf{R}_{\boldsymbol{\nu}}=\mathbb{E}\left\{\boldsymbol{\nu} \boldsymbol{\nu}^{H}\right\}=\operatorname{tr}\left(\mathbf{R}_{\mathrm{e}}\right) \mathbf{I}_{N_{\top}}+N_{0} \mathbf{I} .
$$




\section{Chanel Statistics Estimation}

Exploiting $L$ instantaneous estimated channel state information (CSI) to average over fading, then the statistics CSI can be computed by:

$$
\begin{aligned}
\widehat{\mathbf{H}}_{\mathrm{d}} & =\frac{1}{L} \sum_{i=1}^{L} \widehat{\mathbf{H}}_{i} \\
\widehat{K} & =\frac{\left\|\widehat{\mathbf{H}}_{\mathrm{d}}\right\|_{F}^{2}}{\frac{1}{L} \sum_{i=1}^{L}\left\|\widehat{\mathbf{H}}_{i}-\widehat{\mathbf{H}}_{\mathrm{d}}\right\|_{F}^{2}} \\
\beta & =\frac{\widehat{K}+1}{L} \sum_{i=1}^{L}\left(\left\|\widehat{\mathbf{H}}_{i}\right\|_{F}^{2}-\left\|\widehat{\mathbf{H}}_{\mathrm{d}}\right\|_{F}^{2}\right) \\
\widehat{\mathbf{R}}_{\top} & =\frac{\widehat{K}+1}{\sqrt{\beta} L} \sum_{i=1}^{L}\left(\widehat{\mathbf{H}}_{i}^{H} \widehat{\mathbf{H}}_{i}-\widehat{\mathbf{H}}_{\mathrm{d}}^{H} \widehat{\mathbf{H}}_{\mathrm{d}}\right) .
\end{aligned}
$$




\section{Simulation Parameter}

\begin{tabular}{|c||c|}
\hline \multicolumn{1}{|c||}{ Scenarios } & A1, B1 \\
\hline Tx antennas & 4 \\
\hline Rx antennas & 4 \\
\hline Training symbols for each data slot & $4 \times 4$ \\
\hline Training Length $(\mathrm{L})$ for SCSI estimate & 10 \\
\hline rank $\left(\mathbf{H}_{\mathrm{d}}\right)$ & $1, N_{\mathrm{T}}$, random \\
\hline Random $(\mathrm{AS}, \mathrm{K})$ samples & 10000 \\
\hline Modulation & QPSK \\
\hline
\end{tabular}




\section{$4 \times 4$ MIMO ZF vs. MMSE precoding for scenario A1, mean values}

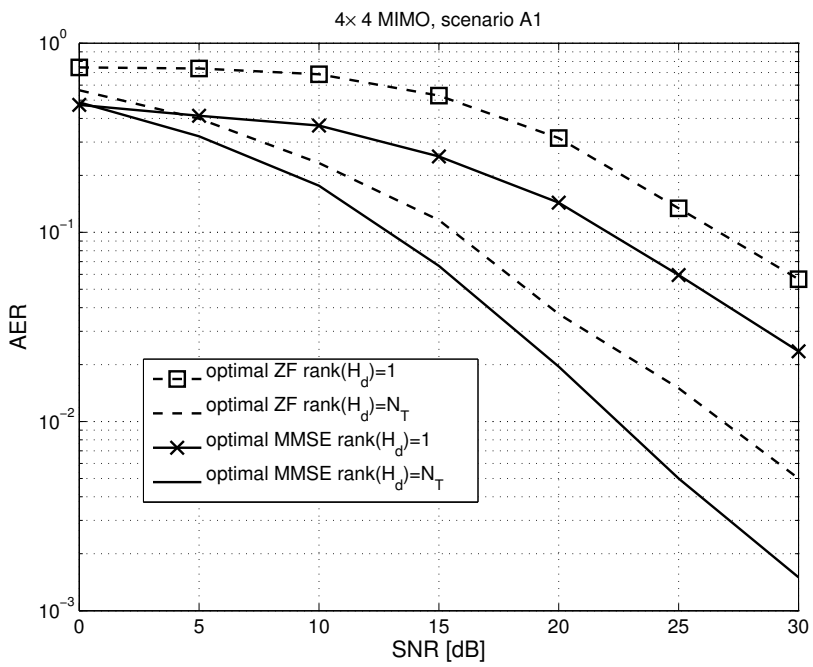




\section{$4 \times 4$ MIMO ZF vs. MMSE precoding for scenario B1, mean values}

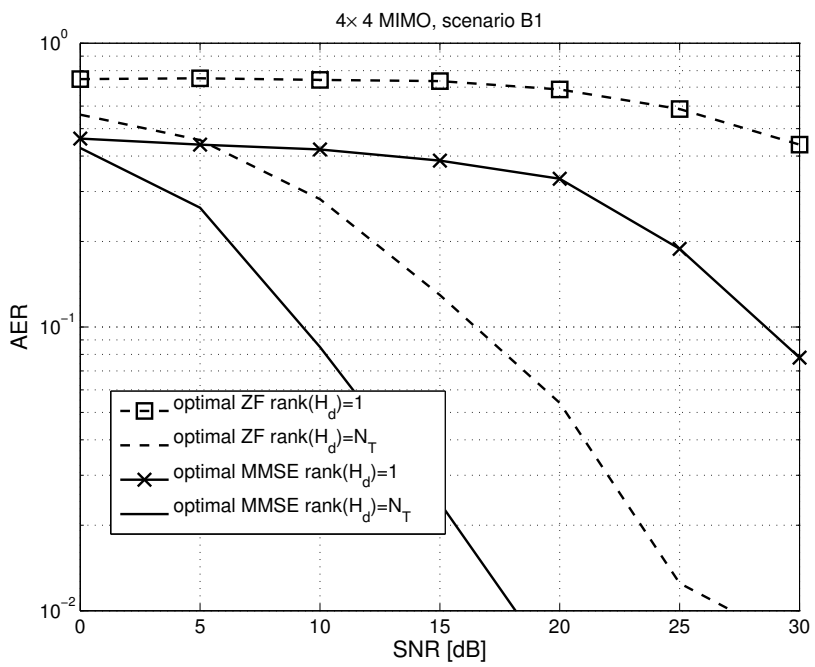




\section{$4 \times 4$ MIMO MMSE precoding for scenario $A 1$, random values}

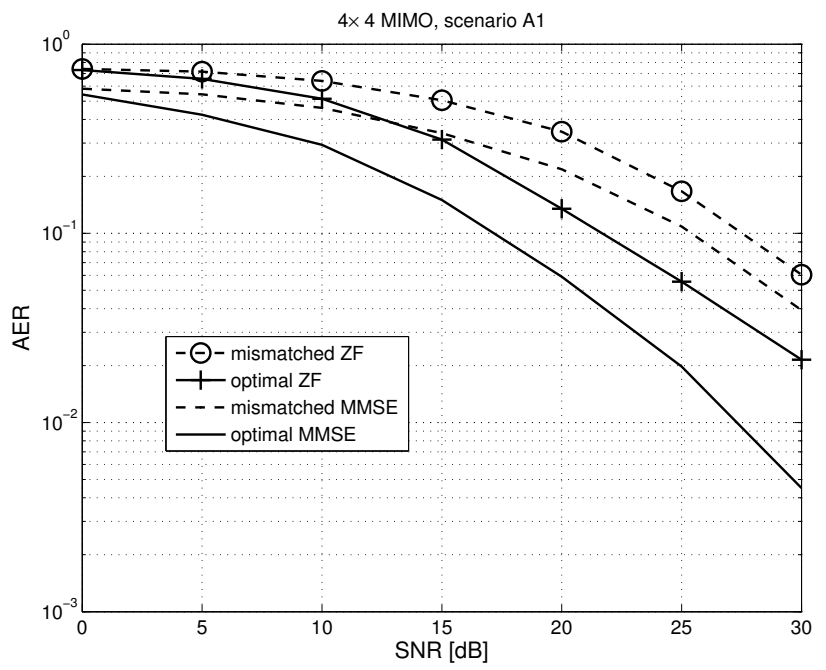




\section{$4 \times 4$ MIMO MMSE precoding for scenario $B 1$, random values}

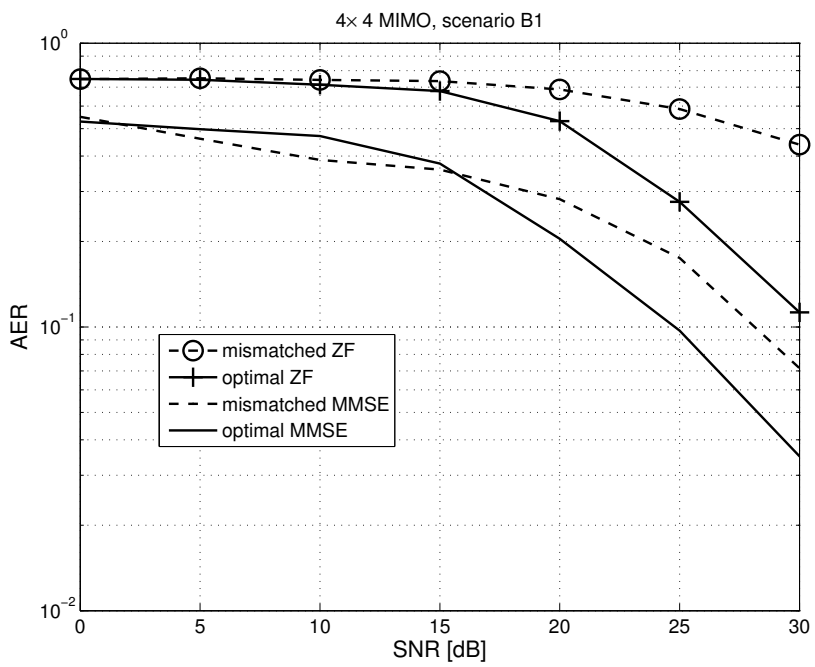


- Evaluated MIMO precoding performance for various assumptions about the channel fading and CSI realistically.

- The performance of precoding can be change significantly due to the different rank

- For the more realistic, i.e., Rician fading channel, the applicable optimal approach accounts for both ICSI and SCSI to transmitter can help improve the precoding performance, comparing with the mismatched approaches

- For less correlated channel, i.e., scenario A1, the optimal ZF precoding can obtain better performance than that of mismatched MMSE precoding. 